\title{
Improving earthquake forecasting by correlations between strong earthquakes and NOAA electron bursts
}

\author{
Cristiano Fidani ${ }^{1,2, *}$ \\ ${ }^{1}$ Osservatorio Sismico “Andrea Bina”, Perugia, Italy \\ ${ }^{2}$ Central Italy Electromagnetic Network, Fermo, Italy
}

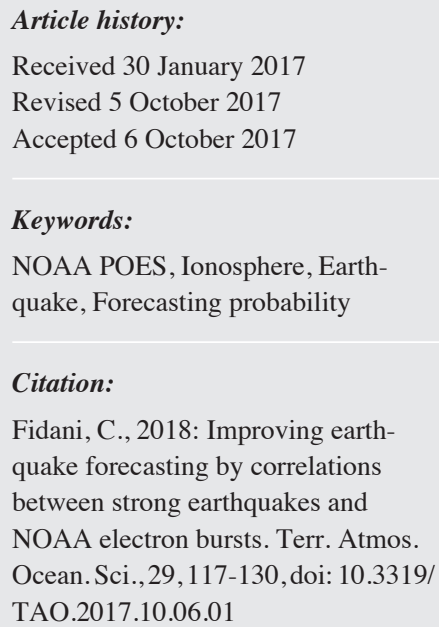
quake forecasting by correlations between strong earthquakes and NOAA electron bursts. Terr. Atmos. Ocean. Sci.,29, 117-130, doi: $10.3319 /$ TAO.2017.10.06.01

\begin{abstract}
NOAA polar orbiting satellite electron flux data have been studied for their time correlations with earthquakes. Electron and proton bursts have also been studied when precipitating into the atmosphere, in order to distinguish correlations with seismic activity from seasonal variations of particle fluxes and solar activity. Data from the dusk/noon NOAA-15 have been analysed using a set of adiabatic coordinates. Specifically, electron and proton data from July 1998 to December 2014 have been compared with nearly 1500 main shocks occurring worldwide during the same period, all with magnitudes greater than or equal to 6 . When considering $30-100 \mathrm{keV}$ precipitating electron bursts, detected by the vertical NOAA-15 telescope and earthquake epicentre projections at altitudes greater than $1400 \mathrm{~km}$, a significant correlation was observed. The electron precipitation excesses were detected $2-3 \mathrm{hrs}$ prior to large seismic events. The stability of this correlation was observed also when considering different electron bursts at each satellite semi-orbit; even if the correlation distribution was no longer a Poissonian. The significance of the correlation peak was evaluated utilising a super-Poissonian distribution. The observation of precipitating electron bursts was used to calculate an increasing probability of strong earthquake occurrence for the Indonesian Region.
\end{abstract}

\section{INTRODUCTION}

Advanced Television Infrared Observation Satellites (TIROS) spacecraft are named after the prototype satellites, TIROS-N, which have flown since 1978 (Davis 2007). The system consists of pairs of satellites, which ensure that every region of the Earth is regularly observed at least twice every $12 \mathrm{hr}$ from about $800 \mathrm{~km}$ altitude. The National Oceanographic and Atmospheric Administration (NOAA) and the National Aeronautics and Space Administration (NASA) have jointly developed a series of Polar Operational Environmental Satellites (POES) for this purpose. Starting with the NOAA-15 POES in 1998, the satellites have had an upgraded version of the Space Environment Monitor (SEM) instruments, the SEM-2. These instruments consist of two sets of detectors that monitor the energetic charged particles both in directional and non-directional ways. These measure fluxes of energetic ions and electrons into the atmosphere

\footnotetext{
* Corresponding author

E-mail:c.fidani@virgilio.it
}

and particle radiation environments on board the Low Earth Orbit (LEO) satellites.

LEO observations have been able to provide physical information on ionospheric and magnetospheric perturbations, possibly caused by electromagnetic (EM) waves. In particular, concerning radiation belt $(\mathrm{RB})$ particle precipitations (Evans et al. 2008), infrared emissions (Yürür 2006), temperature and density variations of the ions and electrons belonging to the ionospheric plasma (Sarkar et al. 2007), and electric and magnetic field fluctuations (Bhattacharya et al. 2009). Particle precipitation fluctuates principally as a result of geomagnetic storms of solar origin, which depend on the temporal response of the magnetosphere plasma to the solar wind speed (Baker 2000). Associations between electron fluxes recorded by NOAA satellites and solar activity have been studied in connection with geomagnetic storms (Obara et al. 2001), under the RBs with $L$-shell, the McIlwain's coordinate (Mcllwain 1966), $L<2$ (Grigoryan et al. 2008) and in the inner RB (even with $L<2$ ) within the South Atlantic Anomaly (SAA) (Asikainen and Mursula 2008). Similar 
studies have been carried out on NOAA proton fluxes, in connection with geomagnetic storms (Søraas et al. 1999), under the RBs (Søraas et al. 2002), and inside them for $L$ $<4$ (Evans et al. 2008). In addition, the ionosphere is influenced by EM emissions of solar flares. Finally, Sudden Ionospheric Disturbances (SID) are caused by X-Ray and ultraviolet ionization, and are followed by particle precipitation (Huang et al. 2007). SID are also produced by Gamma Ray Bursts, as the X-Ray portion of these events will have the same effect as solar flare X-Ray emissions (Mandea and Balasis 2006).

Regarding trapped particles inside the RBs, they follow gyro- and bounce-motion between the hemispheres, as well as longitudinal drift, conserving the adiabatic invariant associated with the periodic motion (Schulz and Lanzerotti 1974), if they are not disturbed. Longitudinal motion of trapped particles is dominated by the energy-dependent gradient-curvature drift (Hudson et al. 2008), and it occurs in opposite directions, for electrons eastwards and positively charged ions westwards, rather than convection. Moreover, longitudinal motion dominates the lower energy ring current (Kivelson and Russell 1995). Specifically, during quiet times, the trapped electrons are distributed into two RBs divided by a slot at $L=2.5$, around which there is relatively low energetic electron flux.

Ionospheric disturbances linked to seismic activity were first observed around the time of the great Alaskan earthquake (EQ) (Davies and Baker 1965) on 28 March 1964. Satellite measurements (Larkina et al. 1983) have confirmed that it provided medium and far field viewing points of lithosphere phenomena with respect to the EQ influence size. Several of the particle detectors used in solar studies from LEO have been used to investigate electron precipitation in connection with strong EQs during geomagnetically quiet times (Sgrigna et al. 2005; Rothkaehl et al. 2006). These studies were based on data coming from satellite missions lasting only a few months or collected a few months with equal attitude data; therein providing weak evidence for correlations (Aleksandrin et al. 2003; Sgrigna et al. 2005). Other observations of enhanced electron fluxes onboard the Intercosmos 24 satellite were detected before the 1990 Iranian earthquake (Bošková et al. 1994). Demeter satellite also reported increases in the particle fluxes at the two closest orbits during local nighttime, above an earthquake having a magnitude of 7.3 , and taking place on $22 \mathrm{No}-$ vember 2004, northeast of New Zealand (Parrot et al. 2006). Electron bursts (EBs) were defined (Sgrigna et al. 2005) as sudden and brief in duration electron counting increases. A criterion for selecting EBs has been applied on high energy electron flux data at $70 \mathrm{keV}-2.34 \mathrm{MeV}$ recorded by IDP on DEMETER (Zhang et al. 2013). Electron fluxes were observed a few days before EQs (Anagnostopoulos et al. 2010), accompanied by broadband $\mathrm{kHz}$ emissions (Anagnostopoulos et al. 2012). The NOAA-15 particle database, which has been collecting data since 1998 , has already been studied for its particle bursts in connection with global seismic activity during quiet solar periods (Fidani and Battiston 2008). This latter analysis reported huge increases in particle fluxes in connection with the largest quakes that struck the Indonesian Region. The inner RB has been reported to be significantly affected by geomagnetic activity, as reported in past studies (Fidani et al. 2010). Magnetic storms can induce sudden electron flux enhancements of more than one order of magnitude near $L=2$, and the particle burst autocorrelations have indicated a clear sun influence also during quiet periods (Fidani et al. 2012). This being so, periods with very low geomagnetic activity were only included in these analyses. Moreover, correlation calculus was carried out using main shocks only, as EQ clustering highly conditioned correlation results (Fidani et al. 2010). Particle bursts were also considered one for each semi-orbit to avoid a multiplicity of correlation events which were correlated with the same EQs, producing a non-Poissonian statistic (Fidani et al. 2010). This analysis reported on huge increases in particle fluxes prior to the largest quakes, which struck the defined Indonesian and Philippines areas, and were statistically correlates with seismic events (Fidani 2014, 2015).

Many EBs for each semi-orbit were analysed in this work, differently from the past publication (Fidani 2015), in order to confirm their correlations with seismic activity. When analysing $30-100 \mathrm{keV}$ precipitating electrons and EQ epicentre projections at altitudes greater than $1400 \mathrm{~km}$, a significant correlation appeared when considering one EB for each semi-orbit, and correlation significance was recalculated. A 2 - $3 \mathrm{hr}$ electron precipitation excess was observed prior to large events in Indonesia and Philippines, as in previous cases, suggesting a $4-10 \mathrm{hr}$ early preparedness of strong EQs influencing the ionosphere (Fidani 2015, 2016a). Based on this correlation, a calculus was proposed to take into account the EB detection by NOAA satellites for strong Indonesian EQ probability (Fidani 2016a, b).

\section{NOAA PARTICLE DATA AND ITS PREPARATION FOR THE ANALYSIS}

The Medium Energy Proton and Electron Detector (MEPED) on board the NOAA satellites is composed of eight solid-state detectors measuring proton and electron counting rates (CRs) from $30 \mathrm{keV}-200 \mathrm{MeV}$ (Evans and Greer 2004), which include the RB populations, energetic solar particle events (protons) and the low energy portion of the galactic cosmic-ray population. The eight detectors consist of two proton telescopes which monitor the CRs in five energy bands having a range of $30 \mathrm{keV}-6.9 \mathrm{MeV}$, two electron telescopes which monitor the CRs in three energy bands in the range $30 \mathrm{keV}-2.5 \mathrm{MeV}$, and four omnidirectional detectors for protons at energies above $16 \mathrm{MeV}$. One telescope views at an angle of $9^{\circ}$, with respect to the local 
zenith. The second telescope views are in the orthogonal direction, along the satellite motion at $90^{\circ}$, with respect to local zenith. The small solid state electron and proton detectors have nominal geometric acceptances of $0.1 \mathrm{~cm}^{2} \mathrm{sr}$ and opening angle apertures of $\pm 15^{\circ}$, which have been recently confirmed by Monte Carlo simulations (Yando et al. 2011). The simulations also suggested the importance of opposite charge contamination effects. In fact, proton contaminations in electron detectors have been excluded only from the lower energy range, taking into account both observations (Asikainen and Mursula 2008) and simulations (Yando et al. 2011). Nonetheless, the electron CRs have been corrected for proton contamination (Rodger et al. 2010) using software downloaded from the Virtual Radiation Belt Observatory (http://virbo.org/POES\#Processing).

Concerning the NOAA archive record (Evans and Greer 2004), it covers a measurement period of $32 \mathrm{sec}$ of data, including a full set of orbital parameters provided every $8 \mathrm{sec}$. In addition, satellite latitude, longitude and altitude data are provided every $2 \mathrm{sec}$. The archive record includes 16 full data collection cycles from the MEPED electron and proton telescope instruments, so they are provided every $2 \mathrm{sec}$ with a sampling interval of $1 \mathrm{sec}$. A selected portion of the SEM2 instrument status, temperature, and system health data as well as data quality and ancillary information, are also included. So that, unreliable CRs have been excluded from the analysis using flags signalling errors in NOAA detectors. Since the energy for the electrons is a cumulative sum over three thresholds equal to $E_{1}=30 \mathrm{keV}, E_{2}=100 \mathrm{keV}$, and $E_{3}=300 \mathrm{keV}$, and the energy interval was determinant for the scope of this analysis to consider the best defined energies, new energy channels derived from the difference of the energy dependent thresholds have been defined for the intervals $30-100,100-300$, and $>300 \mathrm{keV}$. As all sets of orbital parameters were provided at least every $8 \mathrm{sec}$, this value was chosen as the basic time step of the study. Consequently, all other variables were defined in the $8 \mathrm{sec}$ time step. Thus, $8 \mathrm{sec}$ averages of CR, latitude, longitude, MEPED and omnidirectional data were calculated. The first step in the preparation of NOAA data was done with the storage of all binary files into Ntuples (Couet and Goossens 1998) where the time step was $8 \mathrm{sec}$. From 1 July 1998 to 31 December 2014, binary data were downloaded from NOAA (http://www.ngdc.noaa.gov/stp/satellite/poes/dataaccess. html) and examined to exclude uncorrected instrument operations through their corresponding flags.

Inner RB particle flux increases of over one order of magnitude were always observed during the main phase of magnetic storms (Tadokoro et al. 2007), which occurred in rapid response ( $<1$ day) of the inner RB to the solar wind velocity peak (Vassiliadis 2008). SID were considered possible sources of particle flux perturbations as in past discussions (Sgrigna et al. 2005). SID are produced in the ionosphere by enhanced solar radiation during solar flares, which include Very Low Frequency and Low Frequency effects (Deshpande et al. 1972), and cosmic rays (Inan et al. 2007). In order to include the geomagnetic and extraterrestrial influences on the particle fluctuations, the CR data were associated with daily averages of the geomagnetic Ap index and SID (http://www.aavso.org/solar-sids), as well as three hour averages of the Ap index (ftp://ftp.ngdc.noaa. gov/STP/GEOMAGNETICDATA/APSTAR/apindex). The CR exclusions from the correlation analysis occurred when geomagnetic indexes exceeded thresholds, which were calculated by annual and 11-yr sun particle modulations (Fidani et al. 2012). As CR fluctuations originating in the magnetosphere also occur in sub-storm activity, the quality of the selected quiet geomagnetic days was verified including days with Dst variations (http://wdc.kugi.kyoto-u.ac.jp/ dst final/index.html) of less than $30 \mathrm{nT}$.

To correlate seismic activity with NOAA data, a Ntuple was created which contained EQ data including event time, location, magnitude, and depth. The values of the corresponding $L$-shells of the EQ epicentre projected to different altitudes were also calculated using the same methodology that was used for particles and included in the Ntuples. This calculation of the values was performed to determine the possibility of a physical link between EQs and particle fluxes and their space-time locations. The EQ list was downloaded from the Earthquake Center of United States Geological Survey (USGS) at http://neic.usgs.gov/ neis/epic/epic.html, and has been adjusted to eliminate foreshocks and aftershocks.

\section{NOAA-15 ELECTRON DATA ANALYSIS}

The NOAA-15 has been operational since July 1998, on a near sun-synchronous circular orbit (7:30 - 19:30 LT) with a period of $102,98.5^{\circ}$ inclination and an altitude of about $800 \mathrm{~km}$ (Davis 2007). Over this operating time, the satellite LT has been constant for only two years. Nevertheless, it has maintained a dawn/dusk orbit gradually drifting, reaching 4:30 - 16:30 LT in 2012; see horizontal black belts in Fig. 1. Daily averages of CRs have been calculated along the entire satellite orbit, without distinction between semiorbits, as the satellite sun lighting has been nearly the same in both. Starting from the averages, a condition for which CR fluctuations were not likely due to statistical fluctuations with a probability larger than $99 \%$, was defined (Fidani and Battiston 2008). According to previous works (Fidani and Battiston 2008; Fidani et al. 2010, 2012; Fidani 2014, 2015), the daily averages of CRs were calculated in the space of the invariant coordinates. McIlwain (1966) found it convenient to introduce a more geometrically meaningful parameter $L$, which corresponds to the equatorial radius of a drift shell in the case of a dipole field. He extended this definition to non-dipole field distributions, such as the geomagnetic field, by applying the functional relation be- 


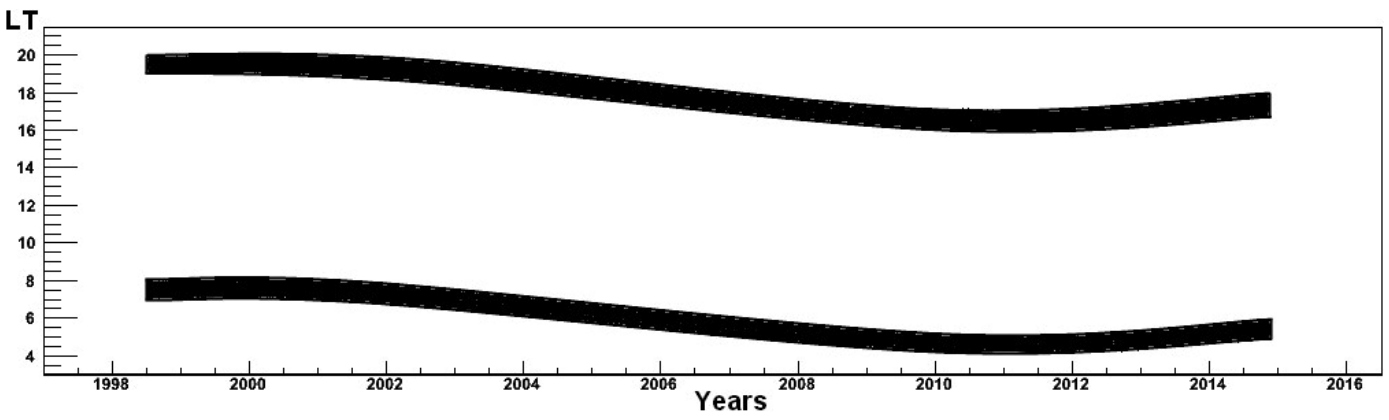

Fig. 1. The local time of the NOAA-15 satellite during the more than $16 \mathrm{yr}$ of this study. Its period, $22 \mathrm{yr}$, coincides with the period of Solar cycle.

tween $L, B_{m}$, and $I$, derived for a dipole field, to non-dipole field distributions. $L$-parameter is defined in terms of the integral: $I=\int_{A}^{A^{\prime}} \sqrt{1-\frac{B(s)}{B_{m}} d s}$, where $A$ and $A^{\prime}$ represent the locations of two magnetically conjugated points, and $B$ and $d s$ are the magnetic field intensity and the line element along the field line respectively. $B_{m}$ is the magnetic field intensity at the points $A$ and $A^{\prime}$. Due to the conservation of the first adiabatic invariant of magnetic moment and of the particle energy $E, B_{m}$ is an adiabatic invariant of motion for all particles mirroring at $A$ and $A^{\prime}$. Due to the conservation of the second invariant of motion $J, I$ also is an adiabatic invariant for all particles mirroring at $A$ and $A^{\prime} . L$ is unambiguously determined for each point on a closed magnetic field line, using $\left(B_{m}, I\right)$ computed with a model magnetic field distribution. Consequently, the coordinate pair $\left(B_{m}, L\right)$ is equivalent to the coordinate pair $\left(B_{m}, I\right)$. Furthermore, NOAA satellite particle detectors look at different directions along their orbits, they will measure different pitch angles $\alpha$ which will be necessary to introduce to describe particle motion as $B_{m}$ $=B / \sin ^{2} \alpha$. The choice of these variables lead to more stable results compared to studies using orbital coordinates, as charged particle motion is strongly variable along the satellite orbit. Together with the $L$-shell and the pitch angle $\alpha$, it was necessary to take into account the CR amplitudes and variations versus geomagnetic coordinates, since the spatial gradient of particle fluxes near the SAA are very large. The geomagnetic field magnitude $B$ can be considered a suitable parameter for delimiting the transition region between inner and outer RBs (Couet and Goossens 1998), where large gradients are located. Intervals of the $B$ value were defined for the analysis, so as to limit the CR amplitude variations. Nonetheless, some regions remained where the particle fluxes and their variations were very high, when the satellite went through the RB in the polar regions and in the SAA. Given this, the SAA was excluded by choosing $B>$ $20.5 \mu \mathrm{T}$, and external RBs were excluded by choosing $L<$ 2.2. Then the averages were calculated for every sector of a three-dimensional matrix formed by the $L$-shell, pitch angle and geomagnetic field intervals. The $L$-shell bin's width was set to 0.1, as in past analyses (Aleksandrin et al. 2003) and the range was chosen between 0.9 and 2.2 to follow
(Fidani and Battiston 2008). The pitch angle was considered equal to the difference between the particle telescope and the geomagnetic field directions. Howeve, the SEM-2 detectors have a finite aperture of $30^{\circ}$, thus a bin width of $15^{\circ}$ was chosen. Finally, the geomagnetic bin width was fixed to be dependent on shorter intervals going through the SAA, because of the necessity to compensate for the non-linear increases in CR when the satellite goes inside this region. The following six $B$ intervals were used to calculate averages: 20.5 - 22.0, 22.0 - 25.0, 25.0 - 28.0, 28.0 - 32.5, 32.5 - 37.0, and $37.0-47.0 \mu \mathrm{T}$, which were slightly different from those of past studies (Fidani et al. 2010) because they had better event fillings.

B-field and $L$-shell were re-evaluated on the NOAA-15 orbit utilising the latest International Geomagnetic Reference Field (IGRF-12) model (http://www.ngdc.noaa.gov/ IAGA/vmod/igrf.html) (Thébault et al. 2015), to include recent model corrections of past years, as well as height variations along the satellite orbits. The IGRF-12 errors for 2015 were expected to be slightly larger than those of previous years; approximately $10 \mathrm{nT}$ (Thébault et al. 2015). Regarding the predictive model, retrospective analysis of previous predictions has shown that errors of up to $20 \mathrm{nT} \mathrm{yr}^{-1}$ are likely (Finlay et al. 2015). In this work, only data from the inner RB, restricting the analysis at $1.0 \leq L \leq 2.2$, was used. It was verified that the errors induced by a $B$ uncertainty of $20 \mathrm{nT}$ on the calculated $L$-shells were always less than 0.05 in the considered subspace. For this, an $L$-shell step of 0.1, defining 12 equal intervals, was utilized. To obtain a reliable statistic, a minimum of 20 satellite passages per day through the same cell $(L, \alpha, B)$ was required (Fidani and Battiston 2008).

In Fig. 2, the satellite positions used in the analysis corresponding to an interval in $(L, \alpha, B)$ space are shown. The multi-coloured area indicates the satellite locations corresponding to observed particle drift precipitation, specifically the violet areas show the satellite geographical positions corresponding to the cells where $B=22.0-25.0 \mu \mathrm{T}$, with $1.1<L<1.2$ and $90^{\circ}<\alpha<105^{\circ}$. Only CRs detected in the violet areas were considered for study perturbations to RBs, as they were drift precipitating particles absorbed into the 


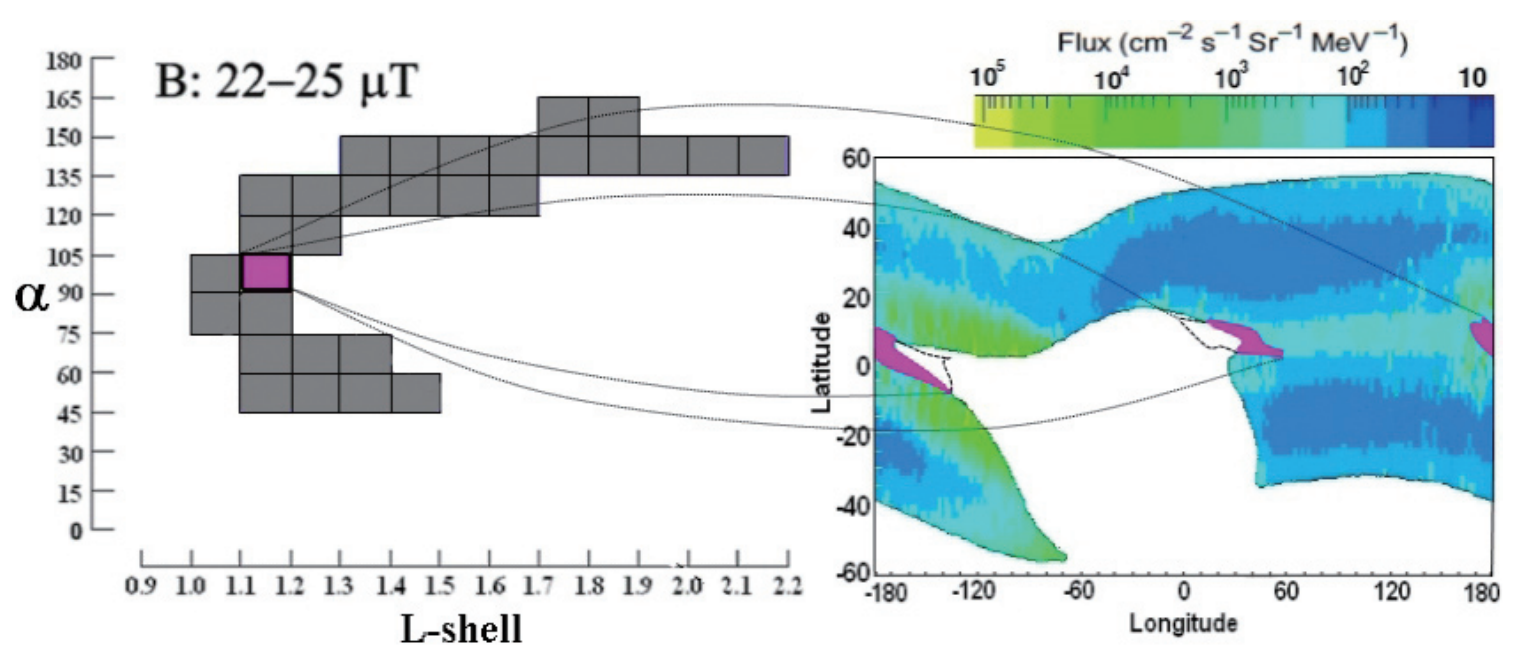

Fig. 2. The data distribution in $L, \alpha$ for a selected $B$-field interval and the corresponding data distribution in latitude and longitude of precipitating electrons, in violet for the precise $(L, \alpha, B)$ interval. On the right, the geographical area considered in this analysis is coloured, while the high flux areas are not. The wave-shaped limits of polar areas are due to the geomagnetic field inclination in relation to the Earth's axis while the birdheadshaped area in the centre represents the inner RB crossing NOAA altitude. Colours indicate the average annual electron flux detected at 30 - $100 \mathrm{keV}$, $0^{\circ}$ NOAA- 15 on board telescope.

atmosphere within the SAA. CR distributions inside such areas were compatible with a Poisson distribution (Fidani and Battiston 2008; Fidani et al.2010). A threshold in sigma was introduced (Fidani and Battiston 2008) so that the amplitude of the CRs exceeded the average value to define the conditions for which a CR was a non-Poissonian fluctuation with $99 \%$ probability. EBs were defined by simply joining contiguous $8 \mathrm{sec}$ non-Poissonian electron CRs, up to a maximum duration of $600 \mathrm{sec}$ (Fidani et al. 2012). Similarly, Proton bursts were defined by simply joining contiguous 8 sec non-Poissonian proton CRs, up to a maximum duration of $600 \mathrm{sec}$. EBs were identical to the contiguous particle bursts defined in (Fidani et al. 2010). Moreover, EB times were defined as the average detection times of the contiguous CRs. CRs were neither stable inside the external RBs because of the influence of variable solar wind, nor inside the internal RB as interactions with electromagnetic waves influenced particle fluxes (Datlowe 2006). The possible influence of EQs on particle fluxes detected by NOAA satellites is easier to determine in a more stable low CR regime. Low CRs were detected outside RBs and CR fluctuations were due to particles leaving the trapping conditions from the inner RB, i.e., precipitating particles. Particle precipitation from the lower boundary of the RBs may have been a result of pitch angle diffusion and drift around the Earth along an $L$-shell (Abel and Thorne 1998). This is due to the fact that along the drift, the altitude of the mirror points varies and when the particles fall below $100 \mathrm{~km}$ they interact with the atmosphere and are lost. UNILIB software (Krunglanski 2003) was used to calculate the minimum $L$-shell bouncing altitudes $h_{\min }$, and consequently particle precipitation events were selected by the condition $h_{\min } \leq 100 \mathrm{~km}$. Annual and 11 yr modulations of EBs are visible in the precipitation plot, relative to the NOAA-15 database, see Fig. 3. This pattern was obtained by summing the daily number of EBs at low $L$-shell intervals, during quiet geomagnetic periods between July 1998 and December 2014 , for the $0^{\circ}$ detector with electron energy from $30-100 \mathrm{keV}$. Figure 3 reports only EBs selected during days with very low geomagnetic activity, while EBs selected during days with medium and high geomagnetic activity were not reported in the same figure. An anticorrelation of EBs number with solar activity appeared. However, the origin of this phenomenon needs further investigation. For example, the presence of high speed solar wind streams ( $\mathrm{Li}$ 2006) was not checked.

Precipitating electrons were concentrated in a fairly small region creating a dovetail-shape, located up until 80 degrees longitude from the westward edge of SAA. This dovetail-shape is green coloured in Fig. 2 right. The precipitating electron flux distribution concentrated westwards of SAA means that bouncing points were near the satellite altitude, just below RB, whereas bouncing points were far above the satellite at the opposite longitudes of SAA, where no electrons can be detected. When lower bouncing points begin to cross the NOAA satellite altitude as longitudes approach SAA, some electrons can be detected. Maximum $\mathrm{CR}$ of non-trapped electrons can be detected immediately west of SAA, where the maximum number of electrons have bouncing altitudes under the satellite orbit and above the atmosphere; see Fig. 4. Bouncing altitudes fall below $100 \mathrm{~km}$ inside the SAA. This latter description can be represented by curved lines, indicating particle mirror points, and straight lines, indicating satellite and atmosphere altitudes, as seen in Fig. 4. 




Fig. 3. Daily sum of precipitating EBs in the drift loss cone over $16.5 \mathrm{yr}$ of NOAA-15 detection in a low $L$-shell interval for electron energy from $30-100 \mathrm{keV}$ of the zenith telescope. The anticorrelation of EBs number with solar activity, which had a minimum between 2005 and 2010 , was obtained considering only the number of EBs when geomagnetic indexes were low.

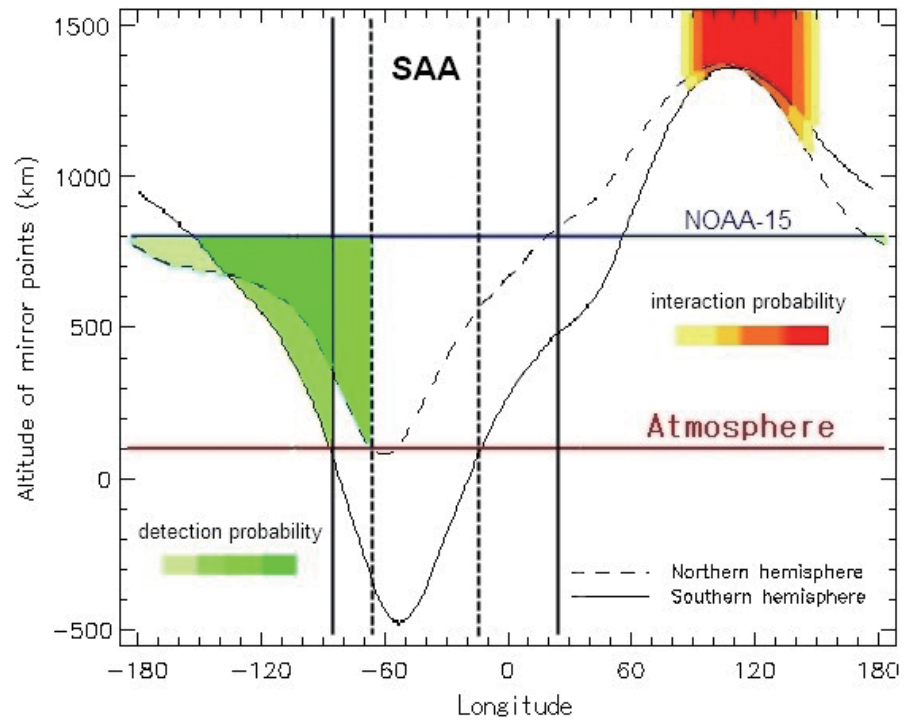

Fig. 4. The altitudes of mirror points for electrons at $L=1.2$ are indicated by continuous and dashed lines and compared to NOAA-15 and atmosphere altitudes, from Fig. 2 of reference (Fidani 2015). Green colour graduated areas indicate altitude/longitude regions where there are bouncing points of electrons that are detectable by NOAA-15 satellite. Red graduated areas are the more frequent areas where interaction between EQs and EBs could occur. The SAA is delimited by vertical lines.

\section{THE CORRELATION BETWEEN EBS AND EQS}

This correlation was calculated using methods from past studies (Fidani and Battiston 2008; Fidani et al. 2010). The calculation started by defining $L_{E Q}$, which was retrieved from the positions of EQ epicentres projected along the vertical at fixed altitudes, so as to evidence some positions in the ionosphere. The corresponding $L$-shells of ionosphere positions were calculated by the same geomagnetic software used for the particle adiabatic calculation. Given that EM resonant wave-particle interactions (Abel and Thorne 1998) may have taken place within the lower boundary altitude of the inner RB, $L$-shells relative to EBs, referred to as $L_{E B}$, were compared with $L$-shell values assigned to each EQ and referred to as $L_{E Q}$. The subsets of EQs and EBs satisfy- ing the condition

$\Delta L=\left|L_{E Q}-L_{E B}\right| \leq 0.1$

was further analysed. Expression Eq. (1) links EQs with all precipitating EBs belonging to well-defined $L$-shells, even if the satellite detects them at different latitudes and longitudes. Condition Eq. (1) is generally satisfied also for all CRs of each EB. This correlation was achieved by filling a histogram with the differences $T_{E Q}-T_{E B}$, between the EQ UT time $T_{E Q}$ and the EB UT time $T_{E B}$, only for those seismic events and particle precipitations which satisfied condition Eq. (1). This calculation was repeated at many altitudes of EQ epicentre projections, by estimating $L_{E Q}$ at each altitude 
from $-600 \mathrm{~km}$ up to $3200 \mathrm{~km}$ in increments of $100 \mathrm{~km}$. A negative altitude for EQs equal to $-600 \mathrm{~km}$ was chosen because $600 \mathrm{~km}$ was the depth of the deepest EQs and some $L$ shells inside the SAA region entered into the earth's crust.

To reduce the effects of solar activity, data corresponding to low values of Ap indexes were considered for 3 and 24-hr intervals (Fidani et al. 2012). Furthermore, EB data were considered "sun influenced" when SID occurred within the same day. Therefore, days when SID occurred were excluded from the correlation. In past works (Fidani et al. 2010), the Ap daily threshold was fixed at 18 , while the three hour Ap indexes were considered with a threshold fixed at 13. Finally, due to long period sun influences which had already been reported in past works, annual and eleven years modulations of the thresholds of two Ap indexes, were also applied to exclude data. The function threshold of geomagnetic indexes was defined based on long term observations:

$\mathrm{Ap}_{m}=f_{m}+g_{m} \sin \left(D_{y}\right)+\left[k_{m}-l_{m} \sin \left(D_{y}\right)\right] \cos \left(D_{s}\right)$

where $m=1$ for 3-hr Ap and $m=2$ for 24-hr Ap, $D_{y}=y$ (year $-y_{0}$ ) and $D_{s}=s$ (day $-s_{0}$ ), $y$ and $s$ are time constants of eleven years sun activity and annual variations respectively. $f_{m}, g_{m}, k_{m}$, and $l_{m}$ are four constants.

The complete NOAA-15 electron CR database, from the beginning of July 1998 to December 2014, was processed and correlated to EQ events over the same period by applying rule Eq. (1). The correlation histogram was calculated without any reference to the EQ magnitudes or EB intensities. Being so, time intervals were filled with unitary weights. Time intervals ranging from a few minutes up to a few days were tested to verify the stability of the $T_{E Q}-T_{E B}$ histogram. A stable correlation histogram was obtained for time intervals around one hour, in agreement with previous studies (Sgrigna et al. 2005). Correlation histograms were studied with respect to: 24-hr Ap, 3-hr Ap, SID, Dst, $L$-shell intervals, CR pitch angle $\alpha$, mirror altitude $h$, EQ magnitude and depth. Solar index modulations were applied with the aims of decreasing solar influence. The optimal modulations were obtained from Eq. (2) with coefficients: $y=$ $0.37, s=0.0172, y_{0}=1996, s_{0}=27, f_{1}=14.8, f_{2}=11.1, g_{1}$ $=1.3, g_{2}=0.8, k_{1}=7.5, k_{2}=2.1, l_{1}=1.3$, and $l_{2}=0.1$. The threshold functions were shown in past publications (Fidani et al. 2012). Even if this modulation corresponds to very small geomagnetic indexes, it was verified that the obtained correlation between EQs and EBs could not be due to substorm events by verifying that in this case Dst variations were always $>-27 \mathrm{nT}$, during the $20 \mathrm{hr}$ before the time of CRs which contributed to the correlation. The analysis was repeated for all the EQ altitude projections.

Several correlation peaks appeared from the first electron energy channels corresponding to $30-100 \mathrm{keV}$ detected by the vertical telescope. Whereas, correlation peaks did not appear from other electron energy channels utilising vertical telescope or from any electron energy channels utilising either horizontal or omnidirectional telescopes. Furthermore, no correlation peaks, to date, have appeared from past NOAA-15 proton analyses. The same algorithm was applied to the particle databases of all of other NOAA polar satellites, but no correlation peaks were observed from the first electron energy channels, nor from any other energy channels or particle types. The dawn-dusk orbit of NOAA15 was the only one different from the other NOAA POES orbits, which were all characterised by day/night.

Correlation plots of $30-100 \mathrm{keV}$ energy channel EBs are shown in Fig. 5, depending on the EQ epicentre projection altitudes. The plot ranges \pm 3 days between EQs and EBs. The distribution shapes and average values calculated on \pm 3 days were compared with standard deviations of correlation events, evidencing super-Poissonian distributions. Significant correlation peaks appeared between 2 and $3 \mathrm{hr}$ of positive time difference, which means that the EB was observed before the corresponding EQ. The peak started to be significant when considering EQ projections above $1400 \mathrm{~km}$ altitudes, see Fig. 5. The correlation was maximised by using EQ magnitude $\mathrm{M} \geq 6$ only. It was clear why the correlation peak had not been observed in the past work (Fidani et al. 2010), where contiguous EB and super-poissonian distributions were also considered. In fact, in that work projection altitudes were under or equal to $800 \mathrm{~km}$ when correlating with EQ magnitudes greater or equal than 6 , while projection altitudes were greater than $800 \mathrm{~km}$ when correlating with EQ magnitudes greater or equal than 5 . Pitch angle restrictions required that the particles be precipitating, meaning that their values were mostly in the loss cone. Specifically, particles in these EBs concentrated in intervals around $65^{\circ}$ and $135^{\circ}$. Results in Fig. 5 were obtained neglecting pitch angles far from these values, with $30^{\circ} \leq \alpha$ $\leq 80^{\circ}$ and $120^{\circ} \leq \alpha \leq 160^{\circ}$. The EQ depths lower or equal to $200 \mathrm{~km}$ were considered; that is they had to have been close to the surface. These results are identical to those obtained when (Fidani 2015) using semi-orbit EBs, which produced Poissonian distributions for correlation events. Furthermore, as indicated by a Referee, the EBs observed a few hours before an EQ in Fig. 5 were always associated with a minimum, around the time of the EQ. This decrease in the correlation is roughly of the same order or even greater than the EB at low altitudes. This observational feature is consistent with the decrease of the VLF wave and electron precipitation activity reported in previous studies (Němec et al. 2009; Anagnostopoulos et al. 2012). However, significance of such peak was low for all altitude projections.

These correlation distributions, shown in Fig. 5, were super-Poissonian in this case, and were different among each other compared to the ratios between the averages and the standard deviations. Being so, to calculate the probability that the peaks were not random fluctuations, an optimal 

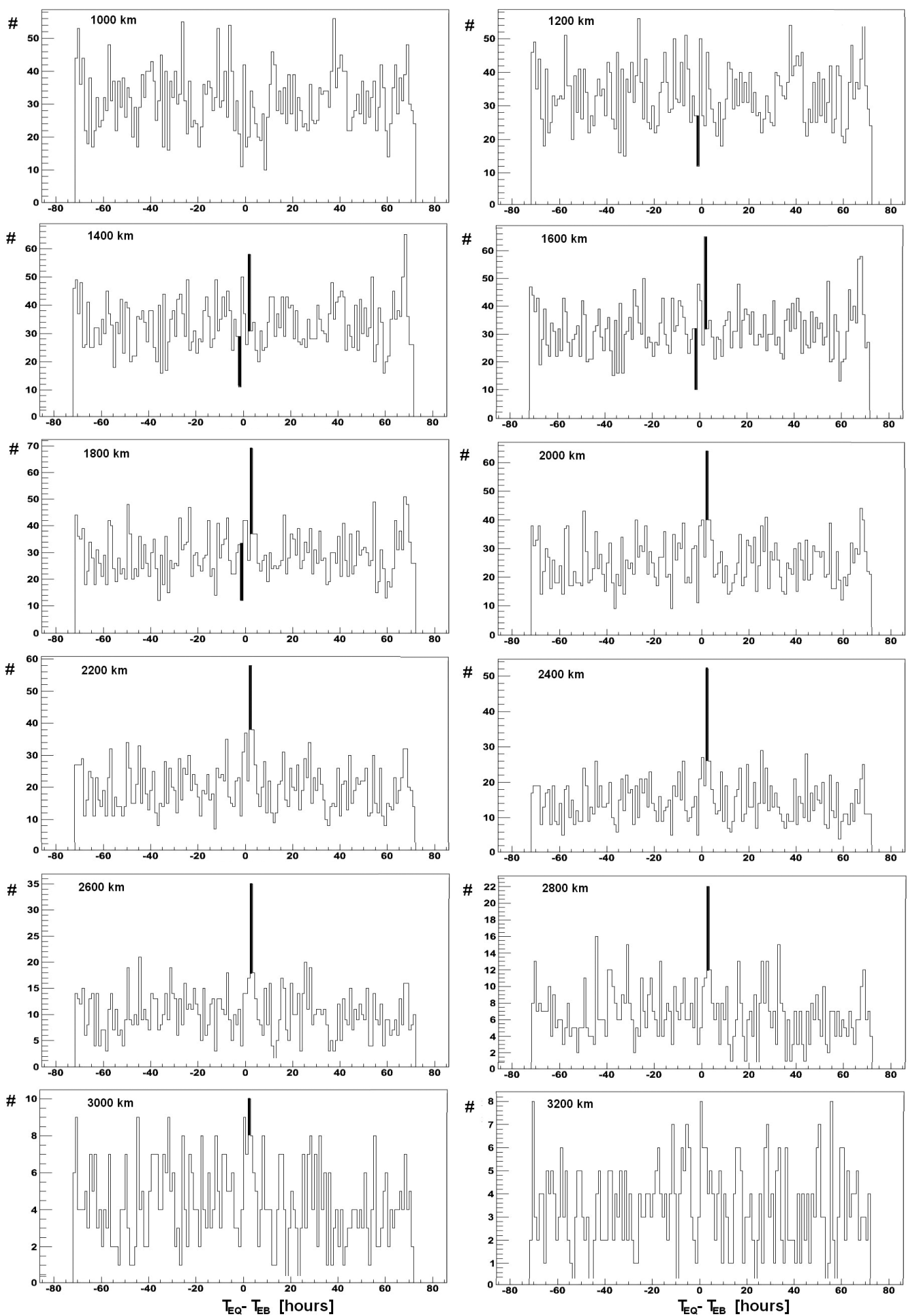

Fig. 5. Correlation plots between EQs and EBs when EQ epicentres were projected at 1000 - 1200 - 1400 - 1600 - 1800 - 2000 - 2200 - 2400 - 2600 - 2800 - $3000-3200 \mathrm{~km}$, the $2-3 \mathrm{hr}$ peak appears for EQ projection altitudes of about $1400 \mathrm{~km}$ and disappears beyond $2800 \mathrm{~km}$. The 2 - $3 \mathrm{hr}$ peak occurs with $35 \mathrm{EQs}$ for projections of $1600 \mathrm{~km}$ and with $14 \mathrm{EQs}$ for projections of $2800 \mathrm{~km}$. Each plot was obtained by summing events of EQs and EBs coincidence occurring with $-72,-71, \ldots, 0, \ldots, 71,72 \mathrm{hr}$ of time differences. Averages were $31-33-33.5-32-29-25.5$ - 20 - 14.5 - 10.5 - 7 4.5 - 3.5 events respectively. Variances were $7.8-8-8.1-7.9-7.5-7-6.2-5.1-4.4-3.6-2.9-2.5$ respectively. The correlation is most significant near $2400 \mathrm{~km}$, where the peak value overcomes the average correlation by about 5 standard deviations. A minimum in the correlation appeared for altitude projections between 1200 and $1800 \mathrm{~km}$. It was of the same magnitude so as the $2-3 \mathrm{hr}$ peak magnitude for altitude projections between 1200 and $1600 \mathrm{~km}$. This minimum correlation peak was between -1 and $-2 \mathrm{hr}$ which means that the minimum occurs when EBs follow between 1 and $2 \mathrm{hr}$ EQs. Correlation peaks were evidenced in black. Significance of the minimum correlation peak was low for all altitude projections. 
function had to be used to approximate correlation distributions. The generalised Poisson distribution (Couet and Goossens 1998) resulted in a very good approximation of correlation distributions. A discrete random variable is said to have a generalised Poisson distribution if its mass function is given by

$$
\begin{aligned}
& P_{n}(\lambda, \theta)=\lambda(\lambda+n \theta)^{n-1} / n ! \exp (-\lambda-n \theta), n=0,1,2, \ldots \\
& P_{n}(\lambda, \theta)=0, n>m, \theta<0
\end{aligned}
$$

and zero otherwise, where $\lambda>0, \max (-1,-\lambda / m) \leq \theta<1$ and $4 \leq m$ is the largest positive integer for which $\lambda+\theta m>0$ when $\theta$ is negative. $\lambda$ and $\theta$ could be obtained from the expressions of the moments (Couet and Goossens 1998), with $\lambda=\left(\tilde{n}^{3} / \Delta n^{2}\right)^{1 / 2}$ and $\theta=1-\left(\tilde{n} / \Delta n^{2}\right)^{1 / 2}$, where $\tilde{n}$ is the correlation average and $\Delta n^{2}$ is the correlation standard deviation. If $N$ is the correlation value corresponding to the peak, the probability that the correlation peak will not be a generalised Poisson fluctuation is $P=\sum_{n=0}^{N-1} P_{n}(\lambda, \theta)$. Being so, the correlation peaks had to be slightly more than 3 standard deviations above the average values to reach a $99 \%$ probability in order not to be generalised Poisson fluctuations. An increase in the statistical significance of the correlation peak was observed with altitude, see Fig. 5. This increase began to exceed 3 standard deviations at $1400 \mathrm{~km}$ and reached a maximum at about $2400 \mathrm{~km}$, where the number of standard deviations was close to 5 . However, the number of correlated events started to decrease for EQ altitude projections above $1400 \mathrm{~km}$ (about 4500) and became very low for altitudes greater than $2400 \mathrm{~km}$ (less than 1500). Both correlation calculations using a randomized space and time distributions of EQs were also checked by using the same EQ times, and the same EQ epicentres, respectively. In all the randomised cases, the previously obtained correlation peaks disappeared. This result was completely similar to those obtained considering semi-orbit EBs (Fidani 2015).

\section{CORRELATION AND INCREASING FORECASTING PROBABILITIES}

The correlation was analysed in time interval resolutions. The minimum considered time interval was $20 \mathrm{~min}$, because this was the maximum duration of detection relative to a single semi-orbit, where the satellite crosses the magnetic conjugate points of a geomagnetic field line. The maximum considered time interval was $3 \mathrm{hr}$, as it was the minimum time definition of the Ap index, which was used to exclude particle data. The calculated correlation resulted stable for all these time intervals. Three correlation plots are reported in Fig. 6, where the time intervals were chosen to be $0.5,1$, and $2 \mathrm{hr}$ in a \pm 1 days analysis, with an EQ altitude projection of $2400 \mathrm{~km}$ and the same conditions of plots, as in Fig. 5. The three plots, reported in Fig. 6, increase the precision of the correlation time: between $2-2.5 \mathrm{hr}$.

Correlation stability, with respect to the considered period length, was also analysed up to \pm 30 days. Figure 7 shows the correlation corresponding to EQ altitude projections of $1800 \mathrm{~km}$. The $2-3 \mathrm{hr}$ peak still dominates the plot, showing it to be the most significant correlation also when


Fig. 6. The correlation peak with EQ projections at $2400 \mathrm{~km}$ was analysed with respect to the time interval to fill the histogram: $0.5,1.0$, and $2.0 \mathrm{hr}$ from left to right, respectively. Correlations were calculated over the $\pm 24 \mathrm{hr}$ interval by summing events of EQs and EBs coincidence occurring with $-24,-23.5, \ldots, 0, \ldots, 23.5,24 \mathrm{hr},-24,-23, \ldots, 0, \ldots, 23,24 \mathrm{hr}$, and $-24,-22, \ldots, 0, \ldots, 22,24 \mathrm{hr}$ of time differences respectively. 


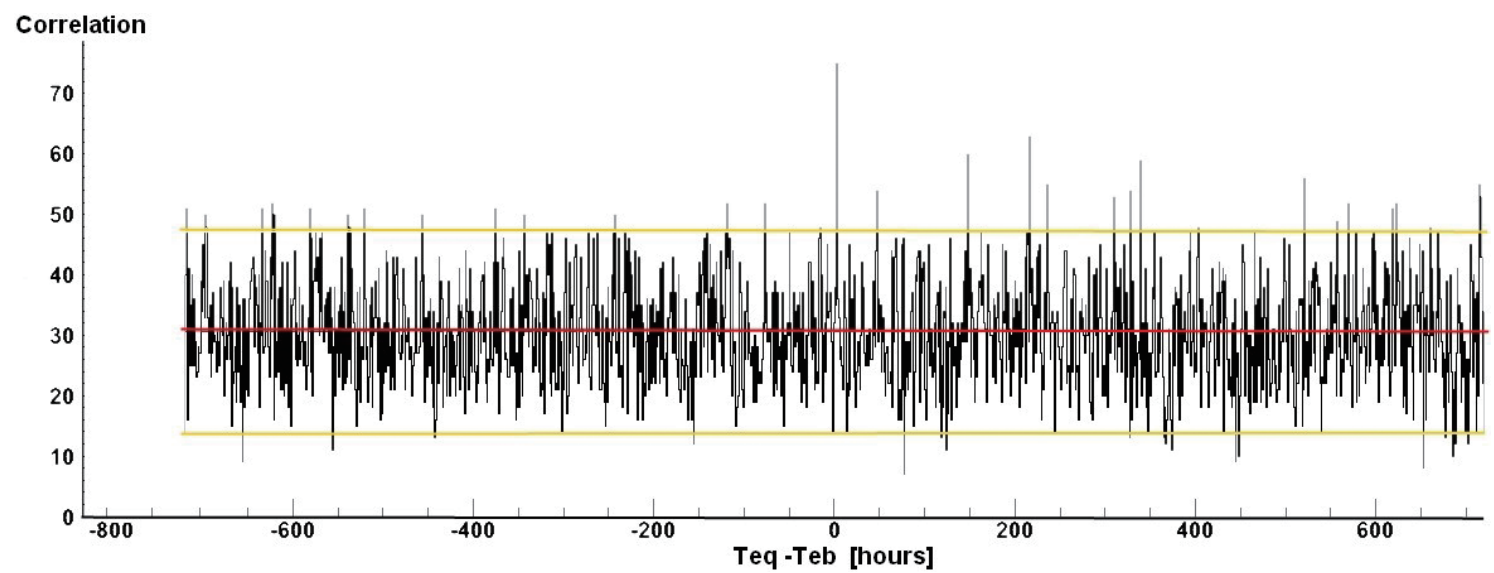

Fig. 7. Correlation plot with EQ projections at $1800 \mathrm{~km}$ calculated over the \pm 30 day interval, the 2 - 3 hr peak remained the main significant correlation. The average was plotted in red and $99 \%$ significance in yellow.

utilising many days of data. Other correlation peaks appear, for example at about 2 days. The significance of such peaks, as all other peaks, is smaller than that of $2-3 \mathrm{hr}$. Increasing the time difference $T_{E Q}-T_{E B}$ up to \pm 150 days, it is possible to see that the $2-3 \mathrm{hr}$ peak is still the main correlation between EQs and EBs corresponding to EQ altitude projections of $1800 \mathrm{~km}$, see Fig. 8. Being so, the observed correlation peak at 2 - $3 \mathrm{hr}$ appears to be stable with respect to both the time binning and to the time extension of the survey. The same results were achieved for all the altitude projections of EQs, wherever the $2-3 \mathrm{hr}$ correlation appears.

Regarding the epicentre locations for EQs correlated with EBs, they were mostly in either the Indonesian or Philippine Regions, with only few events in South America, see Fig. 9. The epicentre positions of all these EQs were principally located in the seas, where strong seismic events more frequently occurred. EQ positions were possibly linked in a causal way to EB positions localised in the dovetail-shape region with some kind of electron disturbances occurring several hour before the main shocks. When perturbations occurred, electron positions were located west of detection positions, as electrons drift eastwards. Disturbances of trapped electrons could have changed both pitch angles and bouncing altitudes in a such way that electrons precipitated into the atmosphere when they reached SAA. Electron drift periods depend on energy according to (Walt 1994):

$\operatorname{Td}=(1.05 / E L)\left[1 /\left(1+0.43 \sin \alpha_{E Q}\right)\right]$

for 30 and $100 \mathrm{keV}$ it proved to be from $21-6 \mathrm{hr}$, respectively. These electrons belonged to the low $L$-shell range of $1.15<L<1.35$, as those obtained for correlations of semiorbit EBs. The electrons which produced the correlation met satellite vertical detectors in the drift loss cone near the SAA about 2 - $3 \mathrm{hr}$ before the EQ time, after having drifted east- wards. This means that the perturbations must have started much earlier. In fact, it was calculated that a further time interval of $2-7 \mathrm{hr}$ was necessary for electrons to cover the $120^{\circ}$ or so, which divided the EQ epicentre longitudes of Sumatra and the Philippines from the dovetail longitudes, see Fig. 9. Then, if disturbances which caused electron precipitations from inner RB occurred above the EQ epicentres in the ionosphere, see Fig. 4 red and orange, they must have anticipated the EQ times by $4-10 \mathrm{hr}$. This was in agreement with the past results from another satellite (Sgrigna et al. 2005), when the correlation between MeV EBs and EQs occurred with a difference of $4-5 \mathrm{hr}$. In fact, the simple expression Eq. (4) produced drift periods of a few minutes for $\mathrm{MeV}$ electrons, and consequently a correlation time difference of $4-5 \mathrm{hr}$ was included into the time interval of $4-10 \mathrm{hr}$, as calculated above.

If such a causal connection exists, a question arises: could the $2-3 \mathrm{hr}$ correlation be used for strong Indonesian EQ forecasting? Following the method developed above to select EBs through adiabatic coordinates, it is currently possible to study EBs in real time, by defining the statistical behaviour of CRs over the $24 \mathrm{hr}$ period preceding the considered time. Starting from here the experiment of Indonesian EQ forecasting would consist of the following phases: (1) the downloading of data immediately after each semi-orbit through the detection area; (2) CR analysis to find EBs; (3) carry out the probability calculation of a strong EQ over the next $2-3 \mathrm{hr}$ in Indonesia or the Philippines.

To carry out phase (1) several ground stations would be necessary to be able to download data from NOAA satellites at the dovetail region corresponding to longitudes between $200^{\circ}$ and $280^{\circ}$. This requirement could be satisfied by the presence of several Northern US ground stations (Evans and Greer 2004). As they are localised in Northern US, downloads of NOAA POES data can occur at the end of up satellite orbits or at the beginning of the next orbit for 


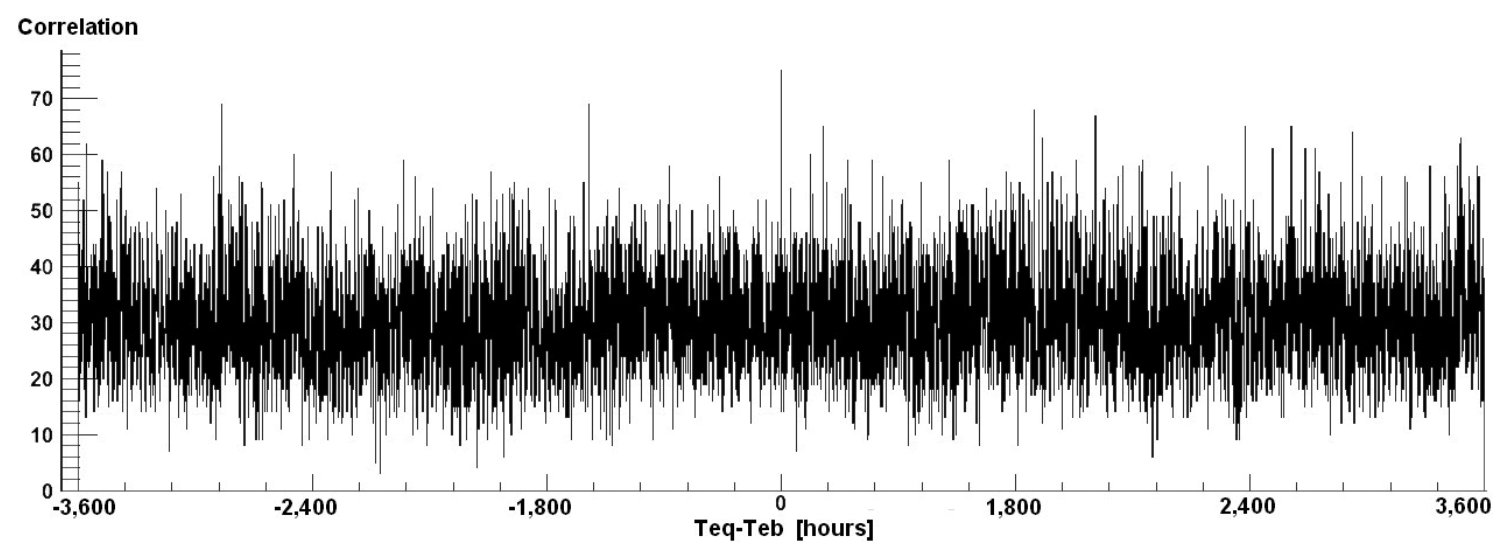

Fig. 8. Correlation plot with EQ projections at $1800 \mathrm{~km}$ calculated over the \pm 150 day interval, the 2 - 3 hr peak remained the main significant correlation. Average and significance were equal to those in Fig. 7.

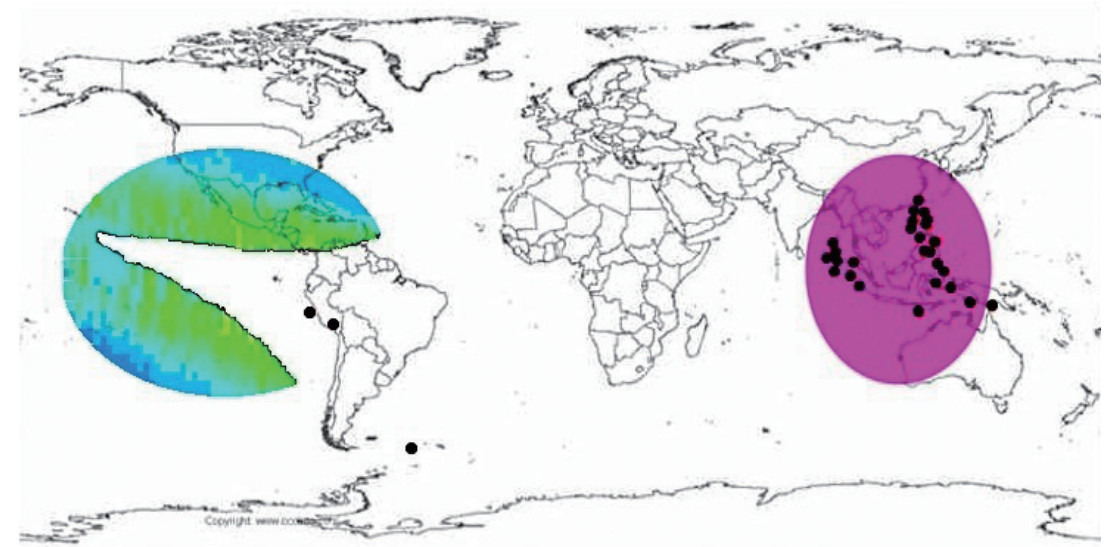

Fig. 9. Epicentres of EQs that contributed to the $2-3 \mathrm{hr}$ correlation are reported here in black; together with the ionospheric region where their preparation influenced the particle motion, which is shown in pink. The dovetail-shape region where EBs were detected and causally related to EQs is shown in blue-green colors.

down orbits. To carry out phase (2), after uploading data in a server, it would be necessary to run automatically the software which realises the steps described above to select EBs. Phase (3) must take into account the results of the statistical correlation of $2-3 \mathrm{hr}$. This can be made through the relation between covariance and correlation (Billingsley 1995) applied to EQ and EB events

$\operatorname{corr}(\mathrm{EQ}, \mathrm{EB})=\frac{\operatorname{cov}(\mathrm{EQ}, \mathrm{EB})}{\sqrt{ } P(\mathrm{EQ})[1-P(\mathrm{EQ})] P(\mathrm{~EB})[1-P(\mathrm{~EB})]}$

with $\operatorname{cov}(\mathrm{EQ}, \mathrm{EB})=[P(\mathrm{EQ} \cap \mathrm{EB})-P(\mathrm{EQ}) P(\mathrm{~EB})]$, and where $P(\mathrm{EQ})$ and $P(\mathrm{~EB})$ are the independent probabilities of EQ and EB occurrence, respectively. Then the joint probability is

$P(\mathrm{EQ} \cap \mathrm{EB})=P(\mathrm{EQ}) P(\mathrm{~EB})+$ $\operatorname{corr}(\mathrm{EQ}, \mathrm{EB}) \sqrt{ } P(\mathrm{EQ})[1-P(\mathrm{EQ})] P(\mathrm{~EB})[1-P(\mathrm{~EB})]$ and the conditional probability $P(\mathrm{EQ} \mid \mathrm{EB})=P(\mathrm{EQ} \cap \mathrm{EB}) /$ $P(\mathrm{~EB})$ is

$P(\mathrm{EQ} \mid \mathrm{EB})=P(\mathrm{EQ})+$

$\operatorname{corr}(\mathrm{EQ}, \mathrm{EB}) \sqrt{ } P(\mathrm{EQ})[1-P(\mathrm{EQ})][1-P(\mathrm{~EB})] / P(\mathrm{~EB})$

This means that, if a correlation exists between EQs and $\mathrm{EBs}$, and the right time difference is considered between $\mathrm{EQ}$ and EB events, the probability of a strong EQ is increased by a term proportional to the correlation.

\section{CONCLUSIONS}

By employing $16.5 \mathrm{yr}$ of NOAA particle data, a statistically significant correlation between EBs and large EQs was confirmed for multiple EBs in a semi-orbit. As in previous results (Fidani 2015) the precipitating electrons occurred at low $L$-shell of $1.1-1.4$ about 2 - $3 \mathrm{hr}$ before main 
shocks. The correlation was very stable with respect to time binning and time extension of the analysis. This result was obtained when EQ depths were less than $200 \mathrm{~km}$, in agreement with other authors (Sgrigna et al. 2005; Němec et al. 2009; Fidani 2015). Unlike past particle precipitation results (Sgrigna et al. 2005), this correlation was observed for both EQs in the sea and in the mainland. Based on the drift period defined as condition Eq. (4), and hypothesizing that a physical interaction between electrons and EQs occurred in the ionosphere, above the epicentre of Sumatra and the Philippine regions, the physical interaction anticipated EQs by $4-10 \mathrm{hr}$. A possibility of using correlation was proposed concerning EQ forecasting, which consists of an evaluation of large Indonesian EQs probability given an EB detection. The conditional probability $P(\mathrm{EQ} \mid \mathrm{EB})$, when a correlation between EQs and EBs exists and time difference is that evidenced by the correlation, increased by a term proportional to the correlation coefficient.

Acknowledgements I would like to thank the "Consulta delle Fondazioni delle Casse di Risparmio Umbre" for supporting this study. Also, I would like to express my thanks to Craig J. Rodger and Janet Green from NOAA for their useful codes to subtract the proton contamination of electron channels. Additionally, I would like to express my gratitude to M. Kruglanski for the computer library to calculate bouncing altitudes.

\section{REFERENCES}

Abel, B. and R. M. Thorne, 1998: Electron scattering loss in Earth's inner magnetosphere: 1. Dominant physical processes. J. Geophys. Res., 103, 2385-2396, doi: 10.1029/97ja02919. [Link]

Aleksandrin, S. Y., A. M. Galper, L. A. Grishantzeva, S. V. Koldashov, L. V. Maslennikov, A. M. Murashov, P. Picozza, V. Sgrigna, and S. A. Voronov, 2003: Highenergy charged particle bursts in the near-Earth space as earthquake precursors. Ann. Geophys., 21, 597-602, doi: 10.5194/angeo-21-597-2003. [Link]

Anagnostopoulos, G., V. Rigas, M. Athanasiou, A. Iliopoulos, E. Vassiliadis, and N. Iossifidis, 2010: Temporal evolution of energetic electron precipitation as a promising tool for earthquake prediction research: Analysis of IDP/DEMETER observations. In: Tsinganos, K., D. Hatzidimitriou, and T. Matsakos (Eds.), Advances in Hellenic Astronomy during the IYA09, ASP Conference Series, Vol. 424, 67-74.

Anagnostopoulos, G. C., E. Vassiliadis, and S. Pulinets, 2012: Characteristics of flux-time profiles, temporal evolution, and spatial distribution of radiation-belt electron precipitation bursts in the upper ionosphere before great and giant earthquakes. Ann. Geophys., 55, 21-36, doi: 10.4401/ag-5365. [Link]
Asikainen, T. and K. Mursula, 2008: Energetic electron flux behavior at low L-shells and its relation to the South Atlantic Anomaly. J. Atmos. Sol.-Terr. Phys., 70, 532538, doi: 10.1016/j.jastp.2007.08.061. [Link]

Baker, D. N., 2000: Effects of the Sun on the Earth's environment. J. Atmos. Sol.-Terr. Phys., 62, 1669-1681, doi: 10.1016/s1364-6826(00)00119-x. [Link]

Bhattacharya, S., S. Sarkar, A. K. Gwal, and M. Parrot, 2009: Electric and magnetic field perturbations recorded by DEMETER satellite before seismic events of the 17th July 2006 M 7.7 earthquake in Indonesia. J. Asian Earth Sci., 34, 634-644, doi: 10.1016/j.jseaes.2008.08.010. [Link]

Billingsley, P., 1995: Probability and Measure (3rd Ed.), John Wiley and Sons, New York.

Bošková, J., J. Šmilauer, P. Tř́íska, and K. Kudela, 1994: Anomalous behaviour of plasma parameters as observed by the intercosmos 24 satellite prior to the iranian earthquake of 20 June 1990. Stud. Geophys. Geod., 38, 213-220, doi: 10.1007/bf02295915. [Link]

Couet, O. and M. Goossens, 1998: HBOOK Statistical Analysis and Histogramming Reference Manual, Information Technology Division, CERN Geneva, Switzerland, $193 \mathrm{pp}$.

Datlowe, D., 2006: Differences between transmitter precipitation peaks and storm injection peaks in low-altitude energetic electron spectra. J. Geophys. Res., 111, doi: 10.1029/2006ja011957. [Link]

Davies, K. and D. M. Baker, 1965: Ionospheric effects observed around the time of the Alaskan earthquake of March 28, 1964. J. Geophys. Res., 70, 2251-2253, doi: 10.1029/jz070i009p02251. [Link]

Davis, G., 2007: History of the NOAA satellite program. $J$. Appl.Remote Sens., 1,012504, doi: 10.1117/1.2642347. [Link]

Deshpande, S. D., C. V. Subrahmanyam, and A. P. Mitra, 1972: Ionospheric effects of solar flares - I. The statistical relationship between X-ray flares and SID's. $J$. Atmos. Sol.-Terr. Phys., 34, 211-227, doi: 10.1016/00219169(72)90165-1. [Link]

Evans, D. S. and M. S. Greer, 2004: Polar Orbiting Environmental Satellite Space Environment Monitor - 2: Instrument Descriptions and Archive Data Documentation. NOAA Technical Memorandum January, version $1.4,155 \mathrm{pp}$.

Evans, D. S., H. Garrett, I. Jun, R. Evans, and J. Chow, 2008: Long-term observations of the trapped high-energy proton population $(\mathrm{L}<4)$ by the NOAA Polar Orbiting Environmental Satellites (POES). Adv. Space Res., 41, 1261-1268, doi: 10.1016/j.asr.2007.11.028. [Link]

Fidani, C., 2014: Positive Correlation between Strong Indonesian and Philippine Region Earthquakes and NOAA Satellite Low L-shell Electron Bursts, $33^{\text {th }}$ Guppo Nazionale di Geofisica della Terra Solida, Bologna, 27-34. 
Fidani, C., 2015: Particle precipitation prior to large earthquakes of both the Sumatra and Philippine Regions: A statistical analysis. J. Asian Earth Sci., 114, 384-392, doi: 10.1016/j.jseaes.2015.06.010. [Link]

Fidani, C., 2016a: Forecasting Strong Earthquakes in Indonesia and Philippines from Space, International Beacon Satellite Symposium BSS-2016, Trieste, Italy.

Fidani, C., 2016b: Strong Earthquakes in Indonesia and Philippines Warned in Advance from Space, 35th General Assembly of the European Seismological Commission ESC-2016, 4-10 September, Trieste, Italy.

Fidani, C. and R. Battiston, 2008: Analysis of NOAA particle data and correlations to seismic activity. Nat. Hazards Earth Syst. Sci., 8, 1277-1291, doi: 10.5194/ nhess-8-1277-2008. [Link]

Fidani, C., R. Battiston, and W. J. Burger, 2010: A study of the correlation between earthquakes and NOAA satellite energetic particle bursts. Remote Sens., 2, 21702184, doi: 10.3390/rs2092170. [Link]

Fidani, C., R. Battiston, W. J. Burger, and L. Conti, 2012: A study of NOAA particle flux sensitivity to solar activity and strategies to search for correlations among satellite data and earthquake phenomena. Int. J. Remote Sens., 33, 4796-4814, doi: 10.1080/01431161.2011.638337. [Link]

Finlay, C. C., N. Olsen, and L. Tøffner-Clausen, 2015: DTU candidate field models for IGRF-12 and the CHAOS-5 geomagnetic field model. Earth Planets Space, 67, doi: 10.1186/s40623-015-0274-3. [Link]

Grigoryan, O. R., M. I. Panasyuk, V. L. Petrov, V. N. Sheveleva, and A. N. Petrov, 2008: Spectral characteristics of electron fluxes at $\mathrm{L}<2$ under the Radiation Belts. Adv. Space Res., 42, 1523-1526, doi: 10.1016/j. asr.2007.12.009. [Link]

Huang, J., Y. Yan, and Y. Liu, 2007: An analysis of solar radio burst events on December 1, 2004. Adv. Space Res., 39, 1439-1444, doi: 10.1016/j.asr.2007.03.061. [Link]

Hudson, M. K., B. T. Kress, H. Mueller, J. A. Zastrow, and J. Bernard Blake, 2008: Relationship of the Van Allen radiation belts to solar wind drivers. J. Atmos. Sol.-Terr. Phys., 70, 708-729, doi: 10.1016/j.jastp.2007.11.003. [Link]

Inan, U. S., N. G. Lehtinen, R. C. Moore, K. Hurley, S. Boggs, D. M. Smith, and G. J. Fishman, 2007: Massive disturbance of the daytime lower ionosphere by the giant $\gamma$-ray flare from magnetar SGR 1806-20. Geophys. Res. Lett., 34, L08103, doi: 10.1029/2006g1029145. [Link]

Kivelson, M. G. and C. T. Russell, 1995: Introduction to Space Physics, Cambridge University Press, New York.

Krunglanski, M., 2003: UNILIB Reference Manual, Belgisch Instituut Voor Ruimte - Aeronomy. Available at
http://www.oma.be/NEEDLE/unilib.php/.

Larkina, V. I., V. V. Migulin, A. V. Nalivaiko, N. I. Gershenzon, M. B. Gokhberg, V. A. Liperovsky, and S. L. Shalimov, 1983: Observations of VLF emission, related with seismic activity, on the Interkosmos-19 satellite. Geomagnetism and Aeronomy, 23, 684-687.

Li, X., 2006: The Role of Radial Transport in Accelerating Radiation Belt Electrons. In: Tsurutani, B., R. McPherron, G. Lu, J. H. A. Sobral, and N. Gopalswamy (Eds.), Recurrent Magnetic Storms: Corotating Solar Wind Streams, American Geophysical Union, Washington, D. C., 139-150, doi: 10.1029/167GM13. [Link]

Mandea, M. and G. Balasis, 2006: The SGR 1806-20 magnetar signature on the Earth's magnetic field. Geophys. J. Int., 167, 586-591, doi: 10.1111/j.1365246x.2006.03125.x. [Link]

McIlwain, C. E., 1966: Magnetic Coordinates. In: McCormac, B. M. (Ed.), Radiation Trapped in the Earth's Magnetic Field. Astrophysics and Space Science Library (A Series of Books on the Developments of Space Science and of General Geophysics and Astrophysics Published in Connection with the Journal Space Science Reviews), Vol. 5, Springer, Dordrecht, 45-61, doi: 10.1007/978-94-010-3553-8_4. [Link]

Němec, F., O. Santolík, and M. Parrot, 2009: Decrease of intensity of ELF/VLF waves observed in the upper ionosphere close to earthquakes: A statistical study. J. Geophys. Res., 114, A04303, doi: 10.1029/2008ja013972. [Link]

Obara, T., Y. Miyoshi, and A. Morioka, 2001: Large enhancement of the outer belt electrons during magnetic storms. Earth Planets Space, 53, 1163-1170, doi: 10.1186/bf03352411. [Link]

Parrot, M., J. J. Berthelier, J. P. Lebreton, J. A. Sauvaud, O. Santolik, and J. Blecki, 2006: Examples of unusual ionospheric observations made by the DEMETER satellite over seismic regions. Phys. Chem. Earth, 31, 486-495, doi: 10.1016/j.pce.2006.02.011. [Link]

Rodger, C. J., M. A. Clilverd, J. C. Green, and M. M. Lam, 2010: Use of POES SEM-2 observations to examine radiation belt dynamics and energetic electron precipitation into the atmosphere. J. Geophys. Res., 115, doi: 10.1029/2008ja014023. [Link]

Rothkaehl, H., R. Bucik, and K. Kudela, 2006: Ionospheric plasma response to the seismic activity. Phys. Chem. Earth, 31, 473-481, doi: 10.1016/j.pce.2006.02.039. [Link]

Sarkar, S., A. K. Gwal, and M. Parrot, 2007: Ionospheric variations observed by the DEMETER satellite in the mid-latitude region during strong earthquakes. $J$. Atmos. Sol.-Terr. Phys., 69, 1524-1540, doi: 10.1016/j. jastp.2007.06.006. [Link]

Schulz, M. and L. J. Lanzerotti, 1974: Particle Diffusion in the Radiation Belts, Springer, Berlin, Heidelberg, doi: 
10.1007/978-3-642-65675-0. [Link]

Sgrigna, V., L. Carota, L. Conti, M. Corsi, A. M. Galper, S. V. Koldashov, A. M. Murashov, P. Picozza, R. Scrimaglio, and L. Stagni, 2005: Correlations between earthquakes and anomalous particle bursts from SAMPEX/ PET satellite observations. J. Atmos. Sol.-Terr. Phys., 67, 1448-1462, doi: 10.1016/j.jastp.2005.07.008. [Link]

Søraas, F., K. Aarsnes, J. Å. Lundblad, and D. S. Evans, 1999: Enhanced pitch angle scattering of protons at mid-latitudes during geomagnetic storms. Phys. Chem. Earth, 24, 287-292, doi: 10.1016/s14641917(98)00041-5. [Link]

Søraas, F., K. Aarsnes, K. Oksavik, and D. S. Evans, 2002: Ring current intensity estimated from low-altitude proton observations. J. Geophys. Res., 107, doi: 10.1029/2001ja000123. [Link]

Tadokoro, H., F. Tsuchiya, Y. Miyoshi, H. Misawa, A. Morioka, and D. S. Evans, 2007: Electron flux enhancement in the inner radiation belt during moderate magnetic storms. Ann. Geophys., 25, 1359-1364, doi: 10.5194/angeo-25-1359-2007. [Link]

Thébault, E., C. C. Finlay, C. D. Beggan, P. Alken, J. Aubert, O. Barrois, F. Bertrand, T. Bondar, A. Boness, L. Brocco, E. Canet, A. Chambodut, A. Chulliat, P. Coïsson, F. Civet, A. Du, A. Fournier, I. Fratter, N. Gillet, B. Hamilton, M. Hamoudi, G. Hulot, T. Jager, M. Korte, W. Kuang, X. Lalanne, B. Langlais, J.M. Léger, V. Lesur, F. J. Lowes, S. Macmillan, M.
Mandea, C. Manoj, S. Maus, N. Olsen, V. Petrov, V. Ridley, M. Rother, T. J. Sabaka, D. Saturnino, R. Schachtschneider, O. Sirol, A. Tangborn, A. Thomson, L. Tøffner-Clausen, P. Vigneron, I. Wardinski, and T. Zvereva, 2015: International Geomagnetic Reference Field: The 12th generation. Earth Planets Space, 67, doi: 10.1186/s40623-015-0228-9. [Link]

Vassiliadis, D., 2008: Response of the radiation belt electron flux to the solar wind velocity: Parameterization by radial distance and energy. J.Atmos. Sol.-Terr. Phys., 70, 1810-1828, doi: 10.1016/j.jastp.2008.05.019. [Link]

Walt, M., 1994: Introduction to Geomagnetically Trapped Radiation, Cambridge University Press, Cambridge, doi: 10.1017/cbo9780511524981. [Link]

Yando, K., R. M. Millan, J. C. Green, and D. S. Evans, 2011: A Monte Carlo simulation of the NOAA POES Medium Energy Proton and Electron Detector instrument. J. Geophys. Res., 116, doi: 10.1029/2011ja016671. [Link]

Yürür, M. T., 2006: The positive temperature anomaly as detected by Landsat TM data in the eastern Marmara Sea (Turkey): Possible link with the 1999 Izmit earthquake. Int. J. Remote Sens., 27, 1205-1218, doi: 10.1080/01431160500212104. [Link]

Zhang, X., C. Fidani, J. Huang, X. Shen, Z. Zeren, and J. Qian, 2013: Burst increases of precipitating electrons recorded by the DEMETER satellite before strong earthquakes. Nat. Hazards Earth Syst. Sci., 13, 197209, doi: 10.5194/nhess-13-197-2013. [Link] 